\title{
Manuel Becker \\ Die Ideokratie als Herrschaftsform - Potentiale eines vergessenen Begriffs in der aktuellen Autokratieforschung
}

Entsprechend ihrer wechselvollen Geschichte und ihrer pluralistischen Ausgestaltungsformen waren die Totalitarismustheorien stets Gegenstand einer breit gefächerten Kritik. Das Kaleidoskop der grundsätzlichen Bedenken gegen den Totalitarismus-Begriff reichte von der Kritik an dessen »normativ-deskriptiver Doppelfunktion « 1 über den Vorwurf einer Relativierung der nationalsozialistischen Verbrechen ${ }^{2}$ bis hin zu der Aussage, die Totalitarismustheorie nehme den »Charakter einer Staatsideologie « ${ }^{3}$ der Bundesrepublik an. Dergleichen fundamentalkritische, primär politisch imprägnierte Einwände dürften den Ansatz aber heute angesichts der entspannteren politischen Situation nach dem Ende des Ost-West-Konflikts im wiedervereinigten Deutschland nicht mehr anfechten. Es wurde auf der anderen Seite ebenso methodisch ernst zu nehmende Kritik an der Totalitarismustheorie geäußert, etwa bezüglich der einseitigen Ausrichtung auf herrschaftsstrukturelle Aspekte, ${ }^{4}$ mit Blick auf die unzureichende Erfassung dynamischer Wandlungsprozesse ${ }^{5}$ oder hinsichtlich der begrenzten empirischen Anwendbarkeit des Konzepts. $^{6}$

Aus der Kritik an den eher statisch konzipierten klassischen Totalitarismustheorien entwickelte sich die moderne Autokratieforschung, die um methodisch offenere und inhaltlich stärker ausdifferenzierte theoretische Modelle von Diktaturen bemüht war. Der wohl einflussreichste und meistrezipierte Beitrag zur modernen Autokratieforschung stammt von Juan Linz. Die verkürzende Dichotomie von totalitären Diktaturen und Demokratien brach er durch eine Binnendifferenzierung von Autokratien in Totalitarismus, Autoritarismus und den häufig vergessenen Sultanismus auf.7 Linz und andere entwickelten verdienstvolle Ansätze, die sich durch interpretative Offenheit und einen

1 Karl Mannheim, Ideologie und Utopie, 3. Aufl., Frankfurt a. M. 1952, S. 232.

2 Vgl. etwa Christian Meier, »Verurteilen und Verstehen. An einem Wendepunkt deutscher Geschichtserinnerung «, in: »Historikerstreit«. Die Dokumentation der Kontroverse um die Einzigartigkeit der nationalsozialistischen Judenvernichtung, München 1987, S. 48-61, S. 53.

3 Wolfgang Wippermann, Totalitarismustheorien. Die Entwicklung der Diskussion von den Anfängen bis beute, Darmstadt 1997, S. 44.

4 Vgl. Hans Mommsen, »Referat «, in: Totalitarismus und Faschismus. Eine wissenschaftliche und politische Begriffskontroverse. Kolloquium im Institut für Zeitgeschichte am 24. November 1978, München 1980, S. 18-27.

5 Vgl. z. B. Chalmers Johnson, Change in Communist Systems, Stanford 1970, S. 2.

6 Vgl. Lothar Fritze, »Unschärfen des Totalitarismusbegriffs. Methodologische Bemerkungen zu Carl Joachim Friedrichs Begriff der totalitären Diktatur «, in: Zeitschrift für Geschichtswissenschaft 43 (1995), S. 629-641, S. 636.

7 Vgl. Juan J. Linz, Totalitäre und autoritäre Regime, 2., überarb. und ergänzte Aufl., Berlin 2003. 
hohen Differenzierungsgrad auszeichnen. Während allerdings der Begriff des Totalitarismus auf zu wenige Diktaturfälle empirisch angewendet werden kann, laboriert der Autokratiebegriff im Gegenteil eher daran, dass die Anwendungspalette heterogen und unübersichtlich ist. ${ }^{8}$ Er bedarf daher der genauen Präzisierung und unterliegt einer permanenten methodischen Herausforderung.

Mit der grundsätzlichen Zurückweisung des klassischen Totalitarismusbegriffs zugunsten eines weiter gefassten Autokratiebegriffs haben einige Autoren gleichsam das Kind mit dem Bade ausgeschüttet. Bereits Linz verzichtete bewusst auf das Kriterium der Ideologie als Grundlage seiner Typologie. Mitunter wurde vergessen, wie entscheidend die Wirkmächtigkeit von Ideologien für die Analyse eines bestimmten Typs autokratischer Herrschaftssysteme ist. Ziel des vorliegenden Aufsatzes ist es, ein wichtiges Erbe der klassischen Totalitarismustheorie in die aktuelle Autokratieforschung zu integrieren: die Betonung ideologischer Deutungsmuster für eine bestimmte Form von Diktaturen. ${ }^{9}$ Um diesem Vermächtnis der frühen Autoren der Totalitarismustheorie gerecht zu werden, soll im Folgenden ein Begriff rehabilitiert werden, der in der aktuellen Debatte angesichts seines analytischen Potentials noch längst nicht die gebührende Berücksichtigung gefunden hat: die Ideokratie. Die Kernthese der Ausführungen lautet, dass der Totalitarismus-Begriff nach wie vor für die vergleichende Untersuchung von $\mathrm{Na}$ tionalsozialismus und Stalinismus ebenso wie auch in der Transformationsforschung eine ganz entscheidende Rolle spielt, dass allerdings der Ideokratie-Begriff für die Typenbildung in der Autokratieforschung methodisch besser geeignet ist.

Der Wortstamm »-kratie« leitet sich vom griechischen »kratos« ab, was so viel wie Macht oder Herrschaft bedeutet, und in der Herrschaftsformenlehre seit der Antike gern als Suffix zur Benennung verschiedener Herrschaftstypen verwendet wird. Das Präfix »Ideo-« verweist auf das griechische »idea «, das ursprünglich Gestalt oder Form bedeutet und spätestens seit Platon als Idee im Sinne von Urbild verstanden wird. Eine Ideologie bezeichnet daran anknüpfend ein gedanklich zusammengefügtes Ideengebäude. Unter der zusammengesetzten Bezeichnung »Ideokratie« soll im Folgenden eine Diktatur oder Autokratie $^{10}$ verstanden werden, die ihre Existenz maßgeblich unter Berufung auf eine deterministische und monistische Weltanschauung legitimiert. Diese Kurzdefinition wird noch genauer auszuführen sein.

Die nachfolgenden Ausführungen widmen sich zunächst dem für die Ideokratie wichtigen Referenzbegriff der Ideologie. Nach einer knappen begriffsgeschichtlichen Skizze folgen einige analytisch-systematische Betrachtungen (1). Anschließend kann zum Ideokratie-Begriff übergeleitet werden, wobei zunächst dessen Rezeptionsgeschichte von den deutschen Historikern und Rechtswissenschaftlern des 19. Jahrhunderts über die Kommunismusforschung bis hin zu aktuellen Studien nachgezeichnet werden soll (2). Nach-

8 Vgl. Paul Brooker, Non-Democratic Regimes. Theory, Government and Politics, New York 2000, S. 21-22.

9 Vgl. Karl Dietrich Bracher, Zeit der Ideologien. Eine Geschichte des politischen Denkens im 20. Jabrbundert, Stuttgart 1982.

10 In den vorliegenden Ausführungen werden die Begriffe Diktatur und Autokratie synonym verwendet. 
folgend werden das analytische Potential sowie einige wesentliche Vorzüge des Ideokratie-Konzeptes gegenüber der klassischen Totalitarismustheorie und anderen Ansätzen der vergleichenden Diktaturforschung herausgearbeitet (3). Danach soll der Ideokratie-Begriff mit Hilfe einer Funktionsanalyse zu einem forschungspraktischen Modell konzeptualisiert werden (4), bevor ein abschließender Blick auf die Grenzen und die Langlebigkeit ideologiegeleiteter Diktaturen geworfen wird (5).

\section{Die Ideologie als Forschungsthema}

Für den Ideologie-Begriff existiert keine einheitliche Definition. ${ }^{11}$ Begriffsgeschichtlich war die Idolenlehre des englischen Renaissancephilosophen Francis Bacon (1561-1626) ein früher gedanklicher Vorläufer. ${ }^{12}$ Für Bacon waren die idola Vorurteile, von denen der Verstand durch die Einsicht in deren Funktionsweise gereinigt werden sollte. Der aus den beiden geistesgeschichtlichen Grundbegriffen »idea« und »logos « zusammengesetzte Terminus wurde erstmals im Jahre 1796 in einem Vortrag des französischen Aufklärers Antoine Louis Claude Destutt, Comte de Tracy (1754-1836) verwendet. ${ }^{13}$ De Tracy war sich durchaus bewusst, einen Neologismus geschaffen zu haben. Es ging ihm und den anderen »Ideologen « der französischen Aufklärung darum, eine neue Wissenschaft der Ideen einzuführen. In seiner Ursprungsbedeutung hatte der Ideologie-Begriff eine dezidiert positive Konnotation. Erst durch Napoleon Bonaparte erhielt der Ideologie-Begriff seine pejorative Einfärbung. Der selbsternannte französische Kaiser verachtete die Ideologen, da sie in seinen Augen realitätsferne Träumer waren, die das nutzlose Räsonieren der politischen Tat vorzogen. ${ }^{14}$ Ihre moderne Bedeutung gewann die »Ideologie« durch Karl Marx. Für Marx kennzeichnete die Ideologen ein idealistischbürgerliches und insofern »falsches Bewusstsein «, demgegenüber das »richtige Bewusstsein « stets dialektisch, materialistisch und proletarisch zu sein hatte. ${ }^{15}$ Marx nutzte den Ideologie-Begriff mit ähnlichen Argumentationsstrukturen wie Bonaparte als polemischen Kampfbegriff gegenüber der Philosophie als bloßer Ideengeschichte. Er setzte ihr unter dem gesellschaftlich-ökonomischen Paradigma die reine Realgeschichte entgegen. Erst mit Lenin setzte sich im Marxismus wieder ein affirmatives Ideologieverständnis

11 In einem assoziativen Verfahren sammelt der britische Kultmarxist Terry Eagelton insgesamt sechzehn verschiedene Verwendungsweisen des Begriffs, ohne Anspruch auf Vollständigkeit $\mathrm{zu}$ erheben oder Hierarchien für diese Liste festlegen zu können. Vgl. Terry Eagelton, Ideologie. Eine Einführung, Stuttgart 1993, S. 7-8.

12 Francis Bacon, Novum organum. Sive indicia vera de interpretatione naturae, Clarendon 1620.

13 Vgl. Antoine Louis Claude Destutt, Comte de Tracy, »Mémoire sur la faculté de penser «, in: Mémoires de l'Institut National des sciences et arts. Sciences Morales et Politiques, Paris 1798, S. 283-450.

14 Vgl. die Sammlung pejorativer Aussagen zum Ideologiebegriff bei Erwin Hölzle, Idee und Ideologie. Eine Zeitkritik aus universalhistorischer Sicht, Bern 1969, S. 135-136.

15 Vgl. Johannes L. Kuppe, »Zur Funktion des Marxismus-Leninismus «, in: Deutscher Bundestag (Hg.), Materialien der Enquête-Kommission Aufarbeitung der Geschichte und Folgen der SEDDiktatur in Deutschland, Bd. III/2, Baden-Baden 1995, S. 1370-1400, S. 1374. 
durch. ${ }^{16}$ Im Nationalsozialismus hingegen wurde der Ideologie-Begriff ganz ähnlich wie bei Napoleon und Marx verachtet und u. a. zur Diffamierung von Intellektuellen verwendet. Unabhängig von der konkreten inhaltlichen Füllung des Begriffs in verschiedenen soziohistorischen Kontexten ging es stets um das auf Marx zurückgehende Wechselverhältnis zwischen Basis und Überbau, zwischen Gedankengebäude und konkreter Wirklichkeit. Soweit der knappe »tour d'horizon« zur Begriffsgeschichte der Ideologie.

Systematische Ansätze zu den Strukturmerkmalen von Ideologien wurden vor allem von den Autoren des Kritischen Rationalismus wie etwa Karl R. Popper, Kurt Salamun oder Ernst Topitsch herausgearbeitet. ${ }^{17}$ Zunächst soll jedoch der eigentliche Begriff genauer präzisiert werden. Roy C. Macridis definiert die Ideologie als »set of ideas and beliefs that people hold about their political regime and its institutions and about their own position and role in it. «18 Macridis Begriffsverständnis steht dabei paradigmatisch für die in der angelsächsischen Literatur dominierende wertneutrale Verwendung der Terminologie, die sowohl Sozialismus und Nationalismus als auch Liberalismus, Konservatismus und Ökologismus als politische Ideologien begreift. ${ }^{19}$ Eine solche Verwendungsweise gründet in der Annahme, dass jedes Denken unvermeidlich ideologiebehaftet sein müsse - ideologiebehaftet insofern, als dass jeder politischen Ordnung, gleich ob sie auf einem dogmatisch geschlossenen Weltbild oder einem liberal offenen Gesellschaftsentwurf aufbaut, gewisse normative Prinzipien, Ideen und Überzeugungen zu Grunde liegen müssen. Diese Auffassung spiegelt auch die einflussreiche Ideologietheorie von Karl Mannheim wider, der zufolge jegliches Denken durch die soziale Existenz und Prägung des denkenden Individuums automatisch vorgeprägt und insofern ideologisch präformiert sei. ${ }^{20}$ Im Hinblick auf die Typenbildung in der Autokratieforschung empfiehlt es sich jedoch, von einem enger gefassten Ideologie-Begriff auszugehen. Mit einem vollkommen abstrahierten und wertneutralen Begriff ließe sich letztendlich jedes politische System als Ideokratie begreifen, womit eine analytische Typologisierung unmöglich würde.

Ein enger konturierter Ideologie-Begriff lässt sich anhand der Differenzierung zwischen Ideologien erster Ordnung und Ideologien zweiter Ordnung, wie sie der Soziologe Eugen Lemberg vorgenommen hat, schärfen. Lemberg versteht unter Ideologien erster Ordnung so genannte Großideologien wie Religionen und politische Weltanschauungen und bezeichnet Ideologien zweiter Ordnung als Ad-hoc-Ideologien, unter denen er Par-

16 Vgl. Eugen Lemberg, Ideologie und Gesellschaft. Eine Theorie der ideologischen Systeme, ibrer Struktur und Funktion, Stuttgart 1971, S. 29.

17 Vgl. Karl R. Popper, Die offene Gesellschaft und ibre Feinde, Bd. 1: Der Zauber Platons, Bd. 2: Falsche Propheten. Hegel, Marx und die Folgen, München 1980; Ernst Topitsch / Kurt Salamun, Ideologie. Herrschaft des Vor-Urteils, München 1972.

18 Vgl. Roy C. Macridis, Contemporary Political Ideologies. Movements and Regimes, 4. Aufl., Glennview 1989.

19 Vgl. exemplarisch auch Robert Eccleshall et al., Political Ideologies. An introduction, Boston 1984; Matthew Festenstein / Michael Kenny, Political Ideologies. A Reader and Guide, Oxford 2005.

20 Vgl. Mannheim, Ideologie und Utopie, aaO. (FN 1). 
tei- und Verbandsprogramme subsummiert. ${ }^{21}$ Diese Überlegung aufnehmend lässt sich die Prämisse formulieren, dass nur Ideologien erster Ordnung im Sinne Lembergs im Herrschaftsgefüge einer Ideokratie Wirksamkeit erlangen können. Damit ist allerdings nur sehr abstrakt und noch nicht hinreichend konkret umrissen, welcher Ideologie-Begriff einer Weltanschauungsdiktatur zu Grunde gelegt werden muss. Daher sollen hier einige Kriterien aufgelistet werden, die eine Ideologie erfüllen muss, um als Grundlage einer diktatorischen Herrschaftslegitimation gelten zu können: (1) Umsetzung: Die Ideologie muss es zur politischen Realisierung in einem autokratischen Regime geschafft haben, d. h. sie muss zum tragenden und politikanleitenden Gedankengerüst eines Herrschaftsgefüges geworden sein. Das unterscheidet sie von einer bloßen politischen Idee, die nie über den Status eines mehr oder weniger verbindenden Elements einer gesellschaftlichen Gruppe mit bestimmten Interessen hinausgekommen ist. (2) Dauerhaftigkeit: Die Ideologie muss über einen bestimmten Zeitraum hin handlungsleitend für Herrschende und loyalitätskonstitutiv für Beherrschte gewirkt haben, damit ihr überhaupt realgeschichtliche Relevanz zugesprochen werden kann. (3) Reichweite: Die Ideologie muss rein quantitativ eine hinreichende Anzahl an Menschen angesprochen und ihr politisches Handeln dominiert haben. (4) Intensität: Sie darf zudem nicht bloß die Rolle einer politisch nachgeordneten oder zu vernachlässigenden Idee einnehmen, sondern sie muss als normatives Fundament vielmehr einen solchen Impetus entwickeln, dass sie absolute Loyalität und bedingungslose Akzeptanz evoziert. (5) Monismus: Das Kriterium Monismus ist der wichtigste und entscheidende Aspekt in Bezug auf die legitimatorische Grundlage ideokratischer Herrschaftssysteme. Alle bisher genannten Kriterien lassen sich auch einem wertneutralen Ideologiebegriff zuordnen. Die Charakterisierung einer Ideologie als monistisch hingegen zielt auf einen bestimmten Ideologiebegriff, der Liberalismus, Konservatismus u. Ä. kategorisch ausschließt. Monismus bezeichnet einen philosophischen Standpunkt, demzufolge alle Prozesse und Phänomene des weltgeschichtlichen Geschehens auf ein singuläres Prinzip oder Gesetz bzw. auf eine zentrale Regel zurückgeführt werden können. Monistische Ideologien können zwar je nach konkreter politischer Situation durchaus flexibel und anpassungsfähig sein, verfügen aber im Kern stets über ein zentrales, unantastbares Dogma. Offenkundige Widersprüchlichkeiten, Doppelbödigkeiten und Brüche des realgeschichtlich Geschehenden werden in ein universales System des vorgeblich »korrekten Denkens « eingeordnet. Monistische Ideologien wähnen sich typischerweise im Besitz allgemeiner Erklärungsschlüssel zum Gang der Weltgeschichte, Abweichungen oder Variationen dieses Erklärungsschlüssels werden entweder verkannt oder nicht geduldet. ${ }^{22}$ Sie bieten zugleich programmatische Vorschriften und praktisch-politische Umsetzungsvorschläge an, um den historischen Fortschritt bestmöglich befördern zu können. Mit diesen Präzisierungen sollte begriffssys-

21 Vgl. Lemberg, Ideologie und Gesellschaft, aaO. (FN 16), S. 40-43.

22 Vgl. Kurt Salamun, »Konfliktverschärfende Tendenzen von Ideologien«, in: Kurt Salamun (Hg.), Ideologie und Aufklärung. Weltanschauungstheorie und Politik, Köln 1988, S. 54-66, S. 58 . 
tematisch hinreichend geklärt sein, welcher Typ von Wert- und Überzeugungssystemen dem hier verwendeten Ideologie-Begriff zu Grunde liegt.

Angesichts der empirischen Vielfalt historisch kontingenter Ideologieausprägungen mangelt es in der Forschung auch nicht an Typologisierungsversuchen: Jaroslaw Piekalkiewicz und Alfred Wayne Penn orientieren ihre Typenbildung anhand der verschiedenen Quellen, aus denen sich die jeweilige Ideologie speist. ${ }^{23}$ Etwas vielschichtiger fällt die ebenfalls vierteilige Typologie von Paul Brooker aus. Er fasst in einer ersten Kategorie Ideologien, die sich auf Nation und Rasse stützen, zusammen und gibt als Beispiele den italienischen Faschismus und den deutschen Nationalsozialismus an. Als zweite Gruppe definiert er Ideologien, die sozialistische Elemente mit adaptierten nationalen oder regionalen Interpretationsmustern mischen und nennt den arabischen und den afrikanischen Sozialismus als Beispiele. Die dritte Untergruppe umfasst Ideologien des kommunistischen Typs wie den Stalinismus und den Maoismus. Schließlich gibt es nach Brooker noch eine vierte, zunächst recht komplex anmutende Gruppe von Ideologien, nämlich solche, die sich auf universelle Prinzipien beziehen und diese mit einem konkreten nationalen Kontext kombinieren. Ein Beispiel hierfür ist etwa der Kemalismus oder die indische Pancasila-Ideologie. ${ }^{24}$ Brookers Typologie erweist sich als sehr differenziert und gut geeignet, um eine große Anzahl an Ideokratien zu erfassen. Es muss allerdings in Rechnung gestellt werden, dass sie nur mit Blick auf die ideologiegeleiteten Einpartei-Diktaturen des 20. Jahrhunderts konzipiert wurde und aus diesem Grund auf Kategorien wie Religion oder kultische Elemente früher Stammesvereinigungen verzichtet.

\section{Die Rezeptionsgeschichte der Ideokratie-Begriffs}

Von diesen analytischen Bemerkungen zum Ideologie-Begriff als Grundlage einer bestimmten Form von Diktatur oder Autokratie soll nun zum Begriff der Ideokratie übergeleitet werden. Auch hier soll ein knapper Blick auf die Rezeptionsgeschichte den begriffssystematischen Präzisionsversuchen vorangestellt werden.

Der Historiker Heinrich Leo (1799-1878) kann als Begriffsschöpfer gelten. ${ }^{25}$ Leo benutzte die Terminologie, um eine Staatsform zu charakterisieren, in der eine »dominierende Ansicht « im Zentrum der Staatslegitimation steht. Das Proprium eines ideokratischen Staates könne folglich nur im Fanatismus bestehen. ${ }^{26}$ Die jüdische Verfassung nach der ersten Unterdrückung durch die Römer und die Jakobinerherrschaft während der Französischen Revolution gelten Leo als Beispiele ideokratischer Herrschaftsformen.

23 Vgl. Jaroslaw Pielkalkiewicz / Alfred Wayne Penn, Politics of Ideocracy, New York 1995, S. 39-41.

24 Vgl. Paul Brooker, Twentieth-Century Dictatorships. The Ideological One-Party States, London 1995, S. 254.

25 Vgl. Ulrich Dierse, »Ideologie«, in: Geschichtliche Grundbegriffe. Historisches Lexikon zur politisch-soziologischen Sprache in Deutschland, Bd. 3, hg. von Otto Brunner et al., Stuttgart 1982, Sp. 131-169, Sp. 143.

26 Heinrich Leo, Zu einer Naturlebre des Staates, Frankfurt a. M. 1948, S. 182.

ZfP 58. Jg. 2/2011 
Während sich der jüdische Staat allerdings auf "göttlicher « Grundlage weiterentwickelt habe, wirke die fanatische jakobinische Ideokratie eher gekünstelt und habe keinen natürlichen Ursprung. Leo hält die Ideokratie in ihrem Kern für eine unreife Staatsform, deren Entstehen in der Regel durch bestimmte Restriktionen begünstigt würde, »die die Befriedigung eines natürlich erwachsenen Staatsbedürfnisses behindern." Ist der Blick auf ein echtes Staatsbedürfnis verstellt, so trete an dessen Stelle eine allumfassende Theorie. Hierin liegt für Leo auch der Grund für das Versiegen ideokratischer Herrschaftsmacht: »Keine Theorie hat eine solche Macht, einen Staat trotz hemmender natürlich erwachsener Verhältnisse zu gründen, es sei denn, sie wäre durch ein wahres Bedürfnis unterstützt. «27

Der Schweizer Rechtswissenschaftler Johann Kaspar Bluntschli (1808-1881) übernahm den Ideokratie-Begriff von Leo in seine Staatsformenlehre. Er unterteilt die verschiedenen Staatsformen in aristotelischer Tradition anhand des Verhältnisses zwischen Regierung und Regierten und dabei insbesondere nach der Herrscherqualität in Ideokratie, Demokratie, Aristokratie und Monarchie. ${ }^{28}$ Bluntschli bezeichnet die Ideokratie als eine der ältesten Staatsformen des Menschengeschlechts. Sie finde sich vorzugsweise in Urzeiten der Geschichte, in denen das menschliche Bedürfnis nach göttlichen Wesen angesichts unverstandener Naturkräfte sehr stark ausgeprägt gewesen sei. Die Ideokratie kennzeichne aus diesem Grund in der Regel ein hoher Einfluss einer priesterlichen Kaste. Als Beispiele werden Äthiopien, Ägypten und das indische Kastensystem genannt. Die Theokratie stellt für Bluntschli die vollkommenste Form der Ideokratie dar. ${ }^{29}$ Besonders interessant ist eine Passage, in der Bluntschli Vor- und Nachteile von Ideokratien gegeneinander abwägt: Als besondere Vorzüge gelten ihm die erhabene herrschaftliche Autorität und die damit verbundene strenge Ordnung; als gravierende Mängel bezeichnet er die inhumane Beziehung zu einem beliebigen Götzen und die unvermeidbare Geringschätzung individueller und politischer Freiheit. Hinzu komme noch, dass eine Ideokratie nicht sonderlich stabil sein könne, da sie auf den fragilen Glauben der Mitglieder an abstrakte Ideen existentiell angewiesen sei. ${ }^{30}$ Dergleichen Ausführungen lesen sich für den modernen Betrachter auffallend scharfsinnig und teilweise sogar prophetisch. Das gilt vor allem für das abschließende Urteil, das Bluntschli über die Ideokratie - wohlgemerkt im Jahre 1852 - fällt: »Todter Buchstabendienst und Formalismus auf der einen, und lügenhafte Sophistik, welche den Schein der Gesetzesherrschaft zu bewahren, in der That aber dasselbe zu umgehen sucht, auf der anderen Seite, stellen sich ein, und im Rahmen des Gesetzes wird halb dem Leben halb dem Rechte Gewalt angethan. « ${ }^{31}$

Auch der Historiker Constantin Frantz (1817-1891) verwendete den Ideokratie-Begriff in seiner Schrift $»$ Die Naturrechtslehre des Staates als Grundlage der Staatswissenschaft « von 1870. In einem Kapitel kontrastiert Frantz theokratische Staatslegitimationen

27 Leo, ebd., S. 186.

28 Vgl. Johann Kaspar Bluntschli, Allgemeines Staatsrecht, geschichtlich begründet, München 1852, S. 156-158.

29 Vgl. Bluntschli, ebd., S. 159-165.

30 Vgl. Bluntschli, ebd., S. 166-167.

31 Bluntschli, ebd., S. 169. 
mit solchen, die auf dem Prinzip der Volkssouveränität gründen. Er polemisiert gegen erstere und verweist alle "göttliche Autorisirung [sic!] der Staatsgewalten in die Fabelwelt«.32 Die Ideokratie ist für Frantz das verblasste Abbild der Theokratie. Als früher Vordenker eines föderativen Staatenbundes in Europa hatte Frantz für theokratische und ideokratische Herrschaftssysteme nur Verachtung übrig.

In der Mitte des 20. Jahrhunderts griff der politische Philiosoph Nicholas Berdyaev (1874-1948) den Ideokratie-Begriff erneut auf. Er benutzte ihn im Rahmen seiner Untersuchungen zur russischen Geistesgeschichte und zu den Ursprüngen des sowjetischen Kommunismus. ${ }^{33}$ Zum gleichen Thema forschten auch Sidney und Beatrice Webb. Sie insistierten, das Sowjetsystem sei mehr als eine bloße Diktatur und stehe in der historischen Tradition von Theokratien und Ideokratien. ${ }^{34}$ Diese Begriffsprägung aus der russischen Kommunismusforschung ist von der klassischen Totalitarismustheorie allerdings weitestgehend ignoriert worden. Lediglich Waldemar Gurian machte sich den Ideokratie-Begriff auf der berühmten von Carl Joachim Friedrich einberufenen Totalitarismuskonferenz von 1953 zu Eigen. In seinen Ausführungen zum Phänomen der »säkularen Religionen « unterschied er Totalitarismen, die um innerweltliche Erlösung bemüht seien, von traditionellen Regimen, die außerweltliche Erlösung in einer postmortalen Existenz in Aussicht stellten. Einer der prominentesten Einwände, der gegen die Charakterisierung totalitärer Bewegungen als »politische Religionen « vorgebracht worden ist, bezieht sich darauf, dass man den Begriff der Religion nicht auf innerweltliche Heilsversprechen beziehen dürfe, sondern dass er ausschließlich für transzendente Bezüge zu reservieren sei. ${ }^{35}$ Gegen diesen Vorwurf führt Gurian den Ideokratie-Begriff ins Feld. Er argumentiert, mit diesem Begriff könne man diejenigen besänftigen, für die es unerträglich sei, den altehrwürdigen Religionsbegriff im Zusammenhang mit den zynischen Manipulationen, unglaublichen Plattitüden und dem terroristischen Schrecken der totalitären Bewegungen anzuwenden. Eine Ideokratie sei in diesem Sinne eine säkularisierte sozio-politische Religion. ${ }^{36}$

Der Ideokratie-Begriff hat ansonsten in der Totalitarismusforschung keine Rolle mehr gespielt. Erst in den 1990er Jahren haben die oben bereits erwähnten Politologen Jaroslaw Piekalkiewicz und Alfred Wayne Penn eine ausführliche Monografie mit dem Titel »Politics of Ideocracy « verfasst, wobei sie den begriffsgeschichtlichen Traditionsstrang aus der deutschen Geschichtsschreibung und Staatrechtslehre im 19. Jahrhundert allerdings nicht aufgreifen. Piekalkiewicz und Penn sehen in solchen Regimen, die ihre Herr-

32 Constantin Frantz, Die Naturlebre des Staates als Grundlage aller Staatswissenschaft, Leipzig 1870, S. 197.

33 Vgl. Nicholas Berdyaev, The Russian Idea, Michigan 1960.

34 Sidney Webb / Beatrice Webb, Soviet Communism. A New Civilization, 2. Aufl., London 1936, S. 450 .

35 Vgl. etwa Hans Maier, »Politische Religionen. Möglichkeiten und Grenzen eines Begriffs«, in: Hans Maier / Michael Schäfer (Hg.), Totalitarismus und politische Religionen. Konzepte des Diktaturvergleichs, Band II, Paderborn et. al. 1997, S. 299-310, S. 306-310.

36 Vgl. Waldemar Gurian, »Totalitarianism and Ideology «, in: Carl J. Friedrich (Hg.), Totalitarianism. Proceedings of a Conference held at the American Academy of Arts and Sciences, Cambridge 1954, S. 119-129, S. 125-126. 
schaftslegitimation aus einer monistischen Ideologie begründen, einen grundlegenden Unterschied zu allen anderen Formen von politischen Systemen. Daher schlagen sie eine neue Typologie politischer Ordnungen vor, die sich aus den beiden Oberkategorien Ideokratie und Pluralismen ableitet. ${ }^{37}$ Auch wenn die Prämisse nur unterstrichen werden kann, so geht der aus ihr abgeleitete Vorschlag dennoch etwas weit. Als Oberkategorien einer umfassenden Herrschaftstypologie scheinen sich nach wie vor die Begriffspaare Demokratie-Diktatur in der Tradition Karl Dietrich Brachers bzw. VerfassungsstaatAutokratie in der Tradition Karl Loewensteins anzubieten; die Ideokratie lässt sich besser und eher als Subtypus eines autokratischen oder diktatorischen Herrschaftstyps einordnen als dass an ihr selbst eine umfassende Herrschaftsformenlehre aufgehangen werden könnte. Ebenso problematisch erscheint die Binnendifferenzierung ideokratischer Herrschaftssysteme bei Piekalkiewicz und Penn: Sie unterscheiden eine totalitäre Form der Ideokratie, die auf der physischen Gewalt gründet, von einer populistischen Variante der Ideokratie, die primär auf den freiwilligen Gehorsam ihrer Beherrschten setzt. ${ }^{38}$ Für den arg konstruiert wirkenden Typus einer "populistischen Ideokratie« dürften sich schwerlich empirische Beispiele finden lassen bzw. es scheint fraglich, ob auf freiwilligen Gehorsam setzende Diktaturen überhaupt sinnvoll unter dem Ideokratie-Begriff rubriziert werden können. Waldemar Gurian aufnehmend ist wohl eine Unterscheidung zwischen religiösen und säkularen Ideokratien gewinnbringender.

In der deutschen Autokratieforschung wird der Ideokratiebegriff von Uwe Backes verwendet, der die Ideokratie als eine von vier Subtypen des autokratischen Herrschaftstyps begreift. Als Subtypen der Ideokratie werden wie bei Gurian die Theokratie auf der Herrschaftsgrundlage einer fundamentalistischen Religion und eine säkulare Ideokratie auf der Basis eines innerweltlichen Heilsversprechens ausdifferenziert. ${ }^{39}$

\section{Das analytische Potential des Ideokratie-Begriffs}

Welche Vorteile hat es nun für die neuere Autokratieforschung, den nahezu vergessenen Terminus Ideokratie wieder aufzunehmen? Welche forschungspraktischen Chancen bietet seine Wiederentdeckung und welche Vorzüge zeichnen ihn im Kontext der Typologisierung autokratischer Regierungssysteme gegenüber dem Totalitarismus-Begriff und dem der "politischen Religion « aus?

Wie eingangs bereits erwähnt, haben einige Autoren trotz der Schwächen verschiedener Totalitarismusmodelle in ihrer allzu harschen Fundamentalkritik das Kind gleichsam mit dem Bade ausgeschüttet. $\mathrm{Zu}$ welchen problematischen Konsequenzen dies teilweise geführt hat, lässt sich am besten anhand der formal-ökonomischen Diktaturmodelle ver-

37 Vgl. Pielkalkiewicz / Penn, Politics of Ideocracy, aaO. (FN 23), S. 20.

38 Vgl. Pielkalkiewicz / Penn, ebd., S. 22.

39 Vgl. Uwe Backes, »Was heißt Totalitarismus? Zur Herrschaftscharakteristik eines extremen Autokratie-Typs «, in: Katarzyna Stoklosa / Andreas Strübind (Hg.), Glaube, Freibeit, Diktatur in Europa und in den USA. Festschrift für Gerhard Besier zum 60. Geburtstag, Göttingen 2007, S. 617-624, S. 617-623; Vgl. auch Uwe Backes, Politische Extreme. Eine Wort- und Begriffsgeschichte von der Antike bis zur Gegenwart, Göttingen 2006, S. 167-188. 
anschaulichen, die sich als eigener Theoriestrang in der Auseinandersetzung mit den klassischen Totalitarismustheorien entwickelt haben. ${ }^{40}$ Diese Modelle gehen in der Regel davon aus, dass der Hauptgrund für die Stabilisierung eines totalitären Herrschaftssystems in der Existenz einer hinreichend großen Anzahl von Regimehörigen zu suchen ist. Ob ein totalitäres Regime überlebt, hängt von den Kosten ab, die es für die Rekrutierung neuer Regimehöriger bzw. für die Elimination nichtintegrierbarer Subjekte zu gewärtigen hat. ${ }^{41}$ Es mag zwar durchaus verdienstvoll für die empirische Anwendung sein, wenn dergleichen Modelle methodisch mit berechenbaren Variablen arbeiten, dennoch beinhalten sie eine offenkundige Fehlinterpretation: Forscher dieses Theoriestrangs neigen dazu, den Diktator oder die Regimeclique ausschließlich als machtbesessene Herrschaftszyniker zu betrachten. In dieser Perspektive degeneriert die Ideologie zu einem bloßen Mittel zum Erhalt und zur Maximierung von Macht. So manchem Ökonomen mag es durchaus schwer einsichtig sein, dass die Verwirklichung einer ideologischen Vision selbst zum politischen Ziel eines diktatorischen Regimes erklärt werden kann. Dies mag damit zusammenhängen, dass eine derart abstrakte Kategorie wie die ideologische Intensität eine nicht quantifizierbare und insofern auch mit mathematisch-quantitativen Methoden nicht operationalisierbare Größe darstellt. Es wäre jedoch verfehlt, diesem Umstand mit dem völligen Ignorieren weltanschaulicher Deutungsmuster zu begegnen. Es kann nicht befriedigen, die Kritik an der Überbetonung des ideologischen Faktors mit der Depravation ideologischer Dogmen zu einem bloßen Machterhaltungsmittel einzulösen. Um diesen Punkt zu konkretisieren: Es besteht ein gewichtiger Unterschied zwischen einer Diktatur wie etwa dem Sanacja-Regime in Josef Pilsudskis Polen (1926-1935) und Regimen wie dem »Dritten Reich«. Was eine einfache, autokratisch geführte Diktatur und ein Regime wie den Nationalsozialismus voneinander unterscheidet, liegt auf der Hand: die monistische Ideologie, aus der sich die Herrschaftslegitimation ableitet. Um den Grad der Herrschaftsintensität, der ein ganz entscheidendes Kriterium zur Klassifikation von Herrschaftstypen ist, angemessen zu erfassen, drängt es sich einer um differenzierte Typenbildung bemühten Autokratieforschung geradezu auf, die Ideologie als Definiens einer ihrer Unterkategorien einzuführen.

Folgt man dieser Annahme, so sollten auch prä- und posttotalitäre Regime im Sinne von Juan J. Linz ${ }^{42}$ als Ideokratien bezeichnet werden. Typusbildend ist in diesem Falle also nicht mehr der Grad der Herrschaftsdurchdringung, sondern die bloße Tatsache, ob sich das betreffende Regime zur Herrschaftslegitimation auf eine monistische Weltanschauung beruft. Ein totalitäres Regime wäre in diesem Fall als extremste Ausprägungsform einer Ideokratie zu begreifen. Das hätte zudem den Vorteil, dass wenig hilfreiche Diskussionen, ob nun beispielsweise die DDR zu einem bestimmten Zeitpunkt noch ein

40 Vgl. exemplarisch Ronald Wintrobe, The political economy of dictatorship, Cambridge 1998; Martin C. McGuire / Mancur Olson, »The Economics of Autocracy and Majority Rule. The invisible hand and the Use of Force", in: Silvio Borner / Martin Paldam (Hg.), The Economic Dimension of Economic Growth, London/New York 1998, S. 38-73.

41 Vgl. Peter Bernholz, »Ideocracy and Totalitarianism. A formal Analysis incorporating ideology «, in: Public Choice 108, Nr. 1-2 (2001), S. 33-75, S. 63.

42 Vgl. Linz, Totalitäre und autoritäre Regime, aaO. (FN 7), S. 222-256.

ZfP 58. Jg. 2/2011 
totalitäres Regime genannt werden sollte, durch nützlichere Leitfragen nach der Art und Weise sowie den Ursachen des Wandels von Ideokratien ersetzt werden könnten. ${ }^{43}$

Eine weitere zumindest fragwürdige Entwicklung eines Teils der modernen Autokratieforschung besteht darin, dass zumeist nur die herrschaftspraktische Seite diktatorischer Herrschaftsgefüge thematisiert wird. Demgegenüber nehmen Fragen der Herrschaftslegitimation eine eher unterprivilegierte Rolle ein. ${ }^{44}$ Auch hier kann es kaum genügen, dass die Bedenken gegenüber einer zu starken Gewichtung des Faktors Ideologie bewusst oder unbewusst in ein pauschales Ausblenden weltanschaulicher Denkmuster münden. Denn es ist gerade für die wissenschaftliche Einordnung und Bewertung einer diktatorischen Herrschaft von entscheidender Bedeutung, nicht nur ihre Symptome, sondern vor allem auch deren tiefer liegende Ursachen in den Blick zu nehmen. Und dies setzt unabdingbar eine ausführliche Beschäftigung mit den normativen Grundlagen der Herrschaft voraus. Insofern könnte sich über den Ideokratie-Begriff auch ein beinahe völlig neues Forschungsfeld eröffnen: nämlich dasjenige der politischen Kultur in Diktaturen. ${ }^{45}$ Der Anwendungsbereich der politischen Kulturforschung war bisher auf Demokratien fokussiert. Ein wertneutrales Begriffsverständnis von »politischer Kultur « schließt jedoch auch ein, dass auch Diktaturen eine politische Kultur haben. Der Faktor Ideologie könnte sich hierbei als fruchtbarer Anknüpfungspunkt erweisen, um Aussagen über das Verhältnis von realem politischen Geschehen und dessen individueller Verarbeitung in der Bevölkerung treffen zu können.

Es lässt sich noch ein weiteres, zugegebenermaßen eher pragmatisches Argument zugunsten des Ideokratie-Begriffs anführen: Unabhängig davon, ob man der grundsätzlichen Kritik an der Kategorie des Totalitarismus zustimmt, kann die Ideokratie vorurteilsfreier verwendet werden. Sie sollte insofern auch für Autoren anschlussfähig sein, die dem Totalitarismus-Begriff aus grundsätzlichen Erwägungen skeptisch gegenüber stehen, ohne dass mit der Betonung des Faktors Ideologie ein wichtiges Erbe der Totalitarismustheorie aufgegeben werden müsste. In diesem Sinne könnte der integrative Charakter des Begriffs als offenes Angebot an die in der Forschung mittlerweile zweifellos marginalisierten Fundamentalkritiker der Totalitarismustheorie verstanden werden. Mit diesem Aspekt ist noch ein weiterer Vorzug des Ideokratie- gegenüber dem Totalitarismus-Begriff verbunden: Während letzterer an den historischen Kontext der neuartigen Diktaturen im Europa des 20. Jahrhunderts gekoppelt ist, stammt ersterer wie oben ausgeführt aus einer sehr viel weiter zurückreichenden Tradition. Auch die angestrengten Versuche, in islamistischen Bewegungen die neuen totalitären Bestrebungen des 21. Jahrhunderts sehen zu wollen, ${ }^{46}$ können nicht verdecken, dass der Begriff des

43 Vgl. Steffen Kailitz, "Stand und Perspektiven der Autokratieforschung «, in: Zeitschrift für Politikwissenschaft 19, Nr. 3 (2009), S. 437-488, S. 447.

44 Vgl. dazu auch Manuel Becker: »Religionsähnliche Züge im Marxismus-Leninismus der DDR. Anmerkungen zu einem Forschungsdesiderat «, in: Deutschland Archiv 1 (2010), S. 127-133.

$45 \mathrm{Vgl}$. zum Zusammenhang von Ideologien und politischer Kulturforschung William T. Bluhm, Ideologies and Attitudes. Modern Political Culture, New Jersey 1974, S. 5-12.

46 Vgl. Bassam Tibi, Der neue Totalitarismus. "Heiliger Krieg" und westliche Sicherheit, Darmstadt 2004. 
Totalitarismus in einer bestimmten historischen Phase entstanden und mit Blick auf bestimmte Formen von Diktatur geprägt worden ist. Ihn unbedacht und gleichsam voreilig auf jede Form extremistischer Bestrebungen ausdehnen zu wollen, hieße auch, ihm seine Konturschärfe und Prägnanz zu nehmen. Der Ideokratie-Begriff steht hingegen in der historischen Tradition der mittelalterlichen Theokratie, wurde bereits lange vor den Totalitarismen des 20. Jahrhunderts in der Herrschaftsformenlehre verwendet und ist insofern zeitlich, örtlich und kulturell deutlich neutraler und analytischer anwendbar.

Begrifflichkeiten, die für die wissenschaftliche Typenbildung geeignet sind, sollten zwei Anforderungen erfüllen: Sie müssen erstens unter einen ordnenden Überbegriff subsumierbar sein, der eine große Bandbreite an empirischen Einzelfällen abdeckt (genus proximum), und sie müssen zweitens eine spezifizierende Bestimmung enthalten, die sie von anderen Unterkategorien unter dem Überbegriff abgrenzt (differentia specifica). Die Ideokratie erfüllt beide Anforderungen: Die Diktatur oder Autokratie lässt sich als plausibles genus proximum der Ideokratie angeben. Die monistische Ideologie wäre als differentia specifica aufzufassen, die die Ideokratie von anderen Diktaturen unterscheidet. Zugleich ist es auf einer weiteren untergeordneten Ebene möglich, zwischen verschiedenen Ideokratietypen zu unterscheiden, etwa zwischen faschistischen Ideokratien, kommunistischen Ideokratien, religiösen Ideokratien etc. In Anlehnung an Gurian bietet sich die Trennung zwischen religiösen und säkular-historizistischen Ideokratien als primäre Differenzierung an. Der Ideokratie-Begriff ist in dieser Hinsicht auch dem Begriff der »politischen Religion « überlegen, dem immer zur Last gelegt worden ist, den Religionsbegriff unpräzise zu benutzen. Der Terminus "politische Religion «, so die Kritik, setze einen falschen Akzent, da die Politik nur als näher bestimmendes Adjektiv auftaucht. ${ }^{47}$ Er passe besser zu islamischen Gottesstaaten, in denen tatsächlich die Religion die Politik absorbiere. Ideologiegeleitete Diktaturen hingegen verführen genau umgekehrt. ${ }^{48}$ Die Analogie zwischen Religion und Ideologie sei ebenfalls nicht so einfach zu unterstellen: Erstens sei die Ideologie in der Regel stärker auf Prinzipien und politische Ziele fokussiert, während sich die allermeisten Religionen lediglich auf unpolitische, geistige Dinge konzentrierten, und zweitens gehe es der Religion um außerweltliche, der Ideologie hingegen um innerweltliche Erlösung. ${ }^{49}$ Die Ideokratie vermag diese Problematik auszuhebeln, da mit ihr sowohl religiöse als auch säkulare Dogmen ohne einseitige Akzentsetzung unter einen Hut gebracht werden können. Entscheidend ist nicht, ob es um ein welttranszendentes oder um ein weltimmanentes Heilsversprechen geht, sondern allein der unverbrüchliche Glaube an monistisch feststehende Dogmen, aus denen die Herrschaftslegitimation abgeleitet wird.

47 Vgl. Philippe Burrin, »Political Religion. The Relevance of a Concept «, in: History and Memory 9, Nr. 1-2 (1997), S. 321-349, S. 326.

48 Vgl. Hans-Günter Hockerts, »War der Nationalsozialismus eine politische Religion? Über Chancen und Grenzen eines Erklärungsmodells«, in: Klaus Hildebrand (Hg.), Zwischen Politik und Religion. Studien zur Entstehung, Existenz und Wirkung des Totalitarismus, München 2003, S. 45-71, S. 68.

49 Vgl. Brooker, Twentieth-Century Dictatorships, aaO. (FN 26), S. 14.

ZfP 58. Jg. 2/2011 
Der Ideokratie-Begriff eignet sich aber nicht nur zur Typologisierung autokratischer Herrschaftssysteme, sondern kann auch gewinnbringend in der vergleichenden Diktaturforschung angewendet werden. Mit einer je nach Untersuchungsgegenstand zu variierenden Erarbeitung der formalstrukturellen Prinzipien kann der Ideokratie-Begriff sinnvoll für die vergleichende Diktaturforschung operationalisiert werden.

\section{Die Funktionen der Ideologie im Herrschaftsgefüge einer Ideokratie}

Angesichts dieser Vielfalt an methodischen Vorzügen, die der Ideokratie-Begriff offenkundig bietet, soll nun ein generelles und abstraktes funktionstheoretisches Modell zur Untersuchung von ideologiegeleiteten Diktaturen entwickelt werden. Eine Funktionsanalyse erfüllt den Zweck, Aussagen über die Herrschaftslogik eines ideokratischen Herrschaftssystems zu treffen, indem sie erhellt, welche Aufgaben die Ideologie in politischer, sozialer und psychologischer Hinsicht zu erfüllen hat. Der Funktionsbegriff wird hierbei nicht politisch-instrumentell aus der Perspektive der Machthaber heraus verstanden, sondern er dient in der Optik des Forschers als analytisches Instrument, um Grundstrukturen des behandelten Gegenstandes offen zu legen. Die verschiedenen von der Forschung bisher entwickelten Funktionskataloge in Bezug auf einzelne Herrschaftssysteme leiden zumeist daran, häufig unvollständig zu sein sowie lediglich enumerativ zu verfahren. ${ }^{50}$ Hier sollen die verschiedenen Funktionen allerdings nicht aufzählend aneinandergereiht, sondern in ein differenzierendes Modell integriert werden, das zunächst von verschiedenen Funktionsbereichen ausgeht, denen in einem zweiten Schritt die einzelnen Funktionen zugeordnet werden.

Hierbei stellt sich zunächst die Frage nach der grundlegenden Unterscheidung, an der die Trennung verschiedener Funktionsbereiche festgemacht werden kann. In diesem Zusammenhang ist auf die bereits erwähnte problematische Verkürzung ideologischer Wirkmacht auf machtpolitische Instrumentalisierung, wie sie etwa viele ökonomische Diktaturmodelle kennzeichnet, zurückzukommen. Es greift nämlich zu kurz, bestimmte Diktatoren oder Herrschaftscliquen lediglich als machtbesessene Machiavellisten anzusehen.

Es ist vielmehr ein typisches Kennzeichen von Ideokratien, dass die Ideologie einerseits einen instrumentellen Charakter annimmt, dass sie aber andererseits auch tatsächlich geglaubt wird. ${ }^{51}$ Dieses Charakteristikum unterscheidet Ideokratien von allen anderen Spielarten autokratischer Herrschaft. Denn instrumenteller Nutzwert und echter Glaube müssen sich nicht zwingend ausschließen, sie können sich im Gegenteil sogar wechselseitig verstärken. Rational kühl kalkulierte Propaganda und irrationaler fanatischer Kult

$50 \mathrm{Vgl}$. exemplarisch in Bezug auf die NS-Ideologie Johannes Zischka, Die NS-Rassenideologie. Machttaktisches Instrument oder handlungsbestimmendes Ideal?, Frankfurt a. M. u. a. 1986, S. 89-105; Vgl. als Beispiel für den Marxismus-Leninismus in der DDR Peter C. Ludz / Ursula Ludz, »Marxismus-Leninismus «, in: DDR-Handbuch, hg. vom Bundesministerium für innerdeutsche Beziehungen, Köln 1985, S. 858-874, S. 873.

51 Lothar Fritze, Verfübrung und Anpassung. Zur Logik der Weltanschaunngsdiktatur, Berlin 2004, S. 35. 
sind in dieser Perspektive zwei Seiten derselben Medaille. Das Wechselverhältnis zwischen machttaktischem Kalkül und überzeugungsbasiertem Dogmatismus eignet sich als Schlüssel zur Einteilung der Funktionsbereiche in dem angestrebten Funktionsmodell. 52

So lässt sich auf der Grundlage der Kategorisierung zunächst ein erster Funktionsbereich (a) definieren, in dem das überzeugungsbasierte Moment dominierend ist. Zu ihm gehören die Orientierungsfunktion (a1), die pseudo-epistemologische Funktion (a2) und die politikanleitende Funktion (a3). Der zweite Funktionsbereich (b) umfasst die Inklusionsfunktion (b1) und die Exklusionsfunktion (b2), die in einem sich wechselseitig ergänzenden Verhältnis zueinander stehen und die sowohl überzeugungsbasiert als auch machttaktisch motiviert sind. Ein dritter Funktionsbereich (c) erfasst schließlich diejenigen Funktionen, in denen tendenziell das machtstrategische Moment den Ausschlag gibt. Ihm lassen sich die edukative Funktion (c1), die Mobilisierungsfunktion (c2) sowie die verschleiernde und kritikimmunisierende Funktion (c3) zuordnen. Eine alle Bereiche übergreifende und zusammenfassende Dimension spiegelt sich in der Legitimationsfunktion (d) wieder. Die folgende schematische Übersicht soll die hier getroffenen Differenzierungen veranschaulichen, bevor die Funktionen im Einzelnen durchdekliniert werden:

\section{Funktionen von Weltanschauungen in Ideokratien}

\begin{tabular}{|c|c|c|}
\hline $\begin{array}{l}\text { (a) primär } \\
\text { überzeugungsbasiert }\end{array}$ & $\begin{array}{l}\text { (b) sowohl überzeugungs- } \\
\text { basiert als auch macht- } \\
\text { taktisch }\end{array}$ & (c) primär machttaktisch \\
\hline (a1) Orientierungsfunktion & (b1) Inklusionsfunktion & (c1) edukative Funktion \\
\hline $\begin{array}{l}\text { (a2) pseudo-epistemologische } \\
\text { Funktion }\end{array}$ & & c2) Mobilisierungsfunktion \\
\hline (a3) politikanleitende Funktion & (b2) Exklusionsfunktion & (c3) Verschleierungsfunktion \\
\hline
\end{tabular}

(d) Legitimationsfunktion

(a) Die wohl offensichtlichste Funktion einer Ideologie ist die weltanschauliche Orientierungsfunktion (a1). Ideologien stellen typischerweise Orientierungsrahmen für das

52 Es handelt sich bei den folgenden Passagen um die Fortentwicklung und Ausarbeitung einer früheren Variante. Vgl. dazu Manuel Becker, Ideologiegeleitete Diktaturen in Deutschland. Zu den weltanschaulichen Grundlagen im „Dritten Reich" und in der DDR, Bonn 2009, S. $174-180$. 
Denken bereit, indem sie dem Individuum in einer komplexen Welt einen gewissen Halt vermitteln. Dies gilt für religiöse wie für säkulare Ideologien gleichermaßen. Der Soziologe Niklas Luhmann hat zwei Aspekte der Orientierungsfunktion ideologischen Denkens besonders betont: zum Einen die Regelungsleistung in Bezug auf die Festlegung einer gesellschaftlichen Wertehierarchie und zum Zweiten im Hinblick auf die Folgen sozialer Aktionen..$^{53}$ Durch die Hierarchisierung von Werten und die Rangordnung von Normen wird ein Denksystem generiert, in dem sich der Einzelne verorten kann. ${ }^{54} \mathrm{Er}$ findet sich so in seine Rolle und dient als kleines Rad in einem großen Getriebe. Nicht selten speisen sich Ideologien dabei aus dem Bewusstsein des historischen Fortschritts, der einen Kulminationspunkt erreicht zu haben scheint, den es für einen fundamentalen Umschwung zu nutzen gilt. In ihrer Orientierungsfunktion wirkt die Ideologie sinnstiftend auf das Bewusstsein des Einzelnen. Weltanschauungen dienen den Menschen gerade in Zeiten gesellschaftlicher Ausdifferenzierungsprozesse und rasanter technischer Entwicklungsschübe als stabilisierendes Gerüst. Simplifizierende Deutungsmuster erleichtern es dem Einzelnen, sich in als unüberschaubar empfundenen Verhältnissen und als bedrohlich angesehenen Entwicklungen besser zu Recht zu finden. Aus diesem Grund schlägt die Stunde von ideokratischen Herrschaftssystemen nicht selten in Krisenzeiten und Perioden gesellschaftlicher Verwerfungen. Allerdings sollte nicht übersehen werden, dass Ideokratien kein offenes Orientierungsangebot bereitstellen, sondern eine geschlossene Form der verordneten Orientierung diktieren.

Mit der Orientierungsfunktion hängt zweitens auch die psendo-epistemologische Funktion (a2) zusammen: Ideologien klären grundsätzliche Seinsfragen des Menschen. ${ }^{55}$ Sie werden als Instrumente zum Verständnis und zur Erklärung der Wirklichkeit, wie sie sich dem Menschen darstellt, in Anspruch genommen. Der Erkenntnisanspruch ist dabei exklusiv. Ideologisches Denken liefert einfache und prima facie schlüssige Antworten auf nahezu alle grundlegenden erkenntnistheoretischen Probleme, die den Menschen beschäftigen. In Wahrheit taugen Ideologien allerdings keinesfalls als Instrument des Wissenserwerbs, da die Resultate aufgrund ihrer monistischen Struktur schon von vorneherein feststehen. Alexander Korchak weist richtigerweise darauf hin, dass eine Ideologie schon von ihrer Natur her gleichsam zur Anti-Wissenschaftlichkeit verdammt ist. Ideologien eignen sich aus Gründen ihrer inneren Beschaffenheit nicht zum Wissenserwerb, da ideologische Aussagen über die Natur, den Menschen und die Welt in der Regel unanfechtbare Glaubensdogmen a priori voraussetzen und sich somit erstens gegen das wissenschaftliche Prinzip der Falsifizierbarkeit verwahren und zweitens keine neuen Erkenntnisse aus ihnen gewonnen werden können. ${ }^{56}$ Das dem jeweiligen Dogmensystem zu Grunde liegende Prinzip, aus dem alle ideologischen Aussagen deduziert werden, ist selbst nicht begründet, sondern wird arbiträr und dezisionistisch

53 Vgl. Niklas Luhmann, »Wahrheit und Ideologie. Vorschläge zur Wiederaufnahme einer Diskussion «, in: Der Staat 1 (1962), S. 431-448, S. 436-439.

54 Vgl. Lemberg, Ideologie und Gesellschaft, aaO. (FN 16), S. 45-46.

55 Vgl. Lemberg, ebd., S. 43-46.

56 Vgl. Alexander Korchak, Contemporary Totalitarianism. A Systems Approach, New York 1994, S. 176. 
festgelegt. Jegliche Empirie wird ausschließlich mit Blick auf das deterministische Grundprinzip des Gedankengebäudes wahrgenommen, wodurch es erst seine innere Geschlossenheit erhält. Aus diesem Grund ist die epistemologische Funktion um das Präfix »pseudo-« zu ergänzen.

Die von der Ideologie bereit gestellten theoretischen Erkenntnisse sollen in die Praxis umgesetzt werden, worin sich die politikanleitende Funktion (a3) ausdrückt. Grunddogmen der Ideologie werden in ideokratisch geprägten Herrschaftssystemen zur Grundlage des politischen Handelns erklärt. Umgekehrt wird Politik aus den weltanschaulich bestimmten Gesetzmäßigkeiten des Lebens abgeleitet. Dies setzt Herrschende wie Beherrschte unter Druck, politische Problemlösungsstrategien als immer im Einklang mit den ideologischen Vorgaben zu rechtfertigen. Das führt nicht selten zu extremen sophistischen Dehnübungen. Wie erklärt es sich zum Beispiel einem Facharbeiter eines volkseigenen Betriebes in einem sowjetischen Satellitenstaat, dass die nicht aus ideologischen Dogmen abgeleiteten betriebswirtschaftlichen Prinzipien der Firmen in den Ländern der EWG offenkundig einen viel höheren Wohlstand erzeugen? Die politischen Steuerungsabsichten in Ideokratien sind in der Regel holistisch angelegt, was mit dem umfassenden und absoluten Erkenntnisanspruch eng zusammenhängt. Aus dem epistemologischen Anspruch auf eine ganzheitliche Erfassung des weltgeschichtlichen Geschehens und des Menschen wird der politische Anspruch auf eine totale Steuerung der Gesellschaft abgeleitet. ${ }^{57}$

Bei der Orientierungs-, der pseudo-epistemologischen und der politikanleitenden Funktion überwiegt jeweils eindeutig der Dogmatismus das Machtkalkül, da diese drei Funktionen überhaupt nur wirksam werden können, wenn sowohl der oder die Machthaber als auch die Bürger oder Untertanen an die ideologischen Dogmen glauben: Beide Seiten müssen davon überzeugt sein, dass die Ideologie einen Rahmen für das Denken vorgeben kann, dass sie erkenntnistheoretisch belastbare Fakten hervorzubringen vermag und dass sie zum Wegweiser für politisches Handeln taugt.

(b) Etwas anders gelagert sind demgegenüber die Funktionen des zweiten Funktionsbereiches. Im Rahmen ihrer Inklusionsfunktion (b1) fördert die Ideologie die Identifikation der Individuen mit dem politischen System. Ideokratien postulieren eine organische soziale Gemeinschaft, in der die Interaktionen auf allen politischen, sozialen, ökonomischen, kulturellen und ethischen Sphären ein integriertes Ganzes ergeben. Politische Homogenität und gesellschaftlicher Kollektivismus kennzeichnen nahezu alle Ideokratien und weisen sie als Repräsentanten einer identitären Gesellschaftskonzeption aus. Im Gegensatz zu pluralistischen Gesellschaftsentwürfen ist der Einzelne nichts ohne das große Ganze und erhält seine Würde erst durch die Zugehörigkeit zum Kollektiv. ${ }^{58}$ Hinzu kommt, dass das Selbstbild in der Regel massiv aufgewertet und überhöht wird. Es muss eine gemeinschaftliche Vision erzeugt werden, die ein Ideal entwickelt, für das

57 Vgl. Armin Pfahl-Traughber, »Gemeinsamkeiten im Denken der Feinde einer offenen Gesellschaft. Strukturmerkmale extremistischer Ideologien «, in: Martin H. Möllers / Robert van Ooyen (Hg.), Jabrbuch öffentliche Sicherbeit 2006/2007, Frankfurt a. M. 2007, S. 21-35, S. 27.

58 Vgl. Popper, Die offene Gesellschaft und ihre Feinde, Bd. 1, aaO. (FN 17), S. 270.

ZfP 58. Jg. 2/2011 
es sich zu kämpfen lohnt. Das setzt ein gehöriges Maß an Realitätsverleugnung oder Realitätsblindheit voraus. Das Herrschaftssystem unter Saparmurat Nijazow in Turkmenistan, um es anhand eines vielleicht weniger prominenten Beispiels zu illustrieren, war in den 1990er Jahren intensiv darum bemüht, tradierte Stammes-Identitäten zurückzudrängen und die historisch nur schwach entwickelte turkmenische Nation als ein autonomes und historisch exzeptionelles Identitätskonzept zu profilieren. ${ }^{59}$ Die Machthaber sind auf die enorme Stabilisierungswirkung der Identifikation des Individuums mit dem ideokratisch geprägten Herrschaftsgefüge existentiell angewiesen. Je abstrakter und wirklichkeitsferner die ideologischen Dogmen konstruiert sind, desto abhängiger ist die Ideokratie von der integrierenden Wirkung ihrer Weltanschauung. Nur ideologiehörige Menschen lehnen vom Regime nicht zugelassene Ideen ab und sind zu deren Bekämpfung bereit. Nur überzeugte Anhänger reagieren stereotyp auf in der Realität auftauchende Widersprüche zum weltanschaulichen Dogma. Zudem eignet sich eine einheitliche und homogene Ideologie propagandistisch hervorragend dazu, den Bürgern bzw. Untertanen ein Gemeinschaftsgefühl zu vermitteln. Daher spielen für die Inklusionsfunktion nicht nur überzeugungsbasierte, sondern ebenso machttaktische Elemente eine Rolle.

Die Hochstilisierung des Eigenbildes hat notwendig die Ausgrenzung des Anderen zur Voraussetzung, womit die Inklusionsfunktion in einem unauflösbaren Zusammenhang mit einer Exklusionsfunktion (b2) steht. Wer sich nicht in die vorgegebenen Dogmen integriert bzw. wer als nicht integrierbar gilt, wird ausgegrenzt und zum Feind erklärt. Feindbildkonstruktionen arbeiten typischerweise mit Sündenbockstrategien. ${ }^{60} \mathrm{Als}$ Sündenböcke an den Pranger gestellte Gesellschaftsgruppen oder Gedankensysteme bieten eine optimale Projektionsfläche, auf der Gefühle wie Wut und Hass ventiliert werden können. Wird dergleichen Verhalten auch noch von offiziell-staatlicher Seite gebilligt und sogar gefördert, so steigt beim Einzelnen auch gleichzeitig das Wertgefühl, etwas Sinnvolles und Nützliches zu tun. Es stellt sich der wohltuende Eindruck ein, auf der »richtigen « Seite zu stehen. Das Freund-Feind-Denken sichert auf diese Weise den Machthabern die Deutungshoheit in Propaganda und Agitation. Dabei ist auffallend, dass Feindbildkonstruktionen in Ideokratien in der Regel auf bipolar-dichotomische Deutungsmuster und damit auf einen Manichäismus hinauslaufen, der keinerlei Schattierungen, Graustufen oder »dritte Wege« zulässt. Ideologiegeleitete Diktaturen betrachten die Gesellschaft stets aus selektiver Perspektive und arbeiten mit hochgradig pseudo-moralisch aufgeladenen Trennungskategorien wie "gut« und »böse«. Es wird suggeriert, der Feind lauere überall, und es bedürfe einer besonderen Wachsamkeit, ihn als solchen zu erkennen. Zu Feindbildern erklärte Gruppen dienen den ideokratischen Machthabern als Beispiele dafür, wie die angeblich fehlgeleiteten Kräfte in der Welt wirken. Sei es der „jüdische Weltbolschewismus“, sei es der „faschistische Kapitalismus“, stets braucht es einen Kontrapunkt, aus dessen Gegensatz eine moralische Ordnung der rechten Gemeinschaft entworfen werden kann. Anhand klar definierter Feindbilder las-

59 Vgl. Slavomir Horak, »The Ideology of the Turkmenbashy Regime«, in: Perspectives on European Politics and Society 6 (2005), S. 305-319.

60 Vgl. Gordon Allport, Die Natur des Vorurteils, Köln 1971, S. 192. 
sen sich auch Gefühle, Meinungen und innere Haltungen illustrieren, die die Regimegläubigen in sich selbst jederzeit zu bekämpfen haben. Gerade das Zusammenspiel von Inklusions- und Exklusionsfunktion scheint eine zentrale Achse im ideologischen Funktionengefüge zu bilden. Im Unterscheid zu den drei voran genannten Funktionen lässt sich hier keine eindeutige Dominanz zugunsten überzeugungsbasierter oder herrschaftsstabilsierender Faktoren angeben. Beide Dimensionen scheinen vielmehr gleichrangig nebeneinander zu rangieren.

(c) Demgegenüber stützen sich die nun folgenden drei Funktionen des dritten Funktionsbereiches primär auf machtzynisch grundierte Motive. In edukativer Funktion (c1) sollen Ideologien erziehend auf die Menschen wirken. In ideokratischen Herrschaftssystemen steht je nach Entwicklungsstand das Erziehungs- und Bildungssystem ganz im Dienste der Diktatur. Die Lehrenden und das Erziehungspersonal werden nach Kriterien der ideologischen Konformität ausgewählt. Texte und Lehrbücher, die nicht den ideologischen Vorgaben entsprechen, werden entweder aussortiert oder im Geiste der Ideologie umgeschrieben. Ein illustratives Beispiel für diesen Aspekt sind die didaktischen Handreichungen der Staatsbürgerkunde-Literatur der DDR. ${ }^{61}$ Machthaber in Ideokratien verfolgen mit der Vereinnahmung des Erziehungs- und Bildungssystems durch die Ideologie gezielte machtpolitische Zwecke. Die Einwirkung auf die Bewusstseinsbildung der Bürger soll sie auf ihre Funktion im gesamtgesellschaftlichen Getriebe vorbereiten. Ideologisches Training gehört zur politischen Sozialisation. Die Menschen sollen von früher Jugend an gefügig gemacht und zu regime- und ideologiehörigen Wesen erzogen werden. Mit Hilfe von durch erzieherische Methoden bereits früh gedanklich gleichgeschalteten Individuen soll ebenso die Fortdauer der Herrschaft für die Zukunft abgesichert werden. Natürlich spielen auch hier überzeugungsbasierte Motivationen eine entscheidende Rolle, in der Herrschaftspraxis dürfte im Verhältnis allerdings eher die machtstrategische Grundierung überwiegen.

Ideologien dienen den ideokratischen Machthabern darüber hinaus dazu, eine mobilisierende Funktion (c2) auf den Bürger bzw. Untertan zu entfalten und motivational auf ihn einzuwirken. Er soll sich durch die in seinem Bewusstsein verankerten ideologischen Dogmen dazu verpflichtet fühlen, sich aktiv für deren Durchsetzung einzusetzen und notfalls mit existentieller Hingabe für sie zu kämpfen. Die Beherrschten sollen für ein Ziel mobilisiert werden, das angeblich im objektiven Interesse aller liegt. Ideokratische Herrschaft ist häufig dadurch gekennzeichnet, dass sie alle Dinge in permanenter Bewegung hält. Diesen Zusammenhang hat bereits Hannah Arendt in ihrer Totalitarismusstudie eindrucksvoll herausgestellt. ${ }^{62}$ Stillstand bedeutet bei der Verwirklichung der ideologischen Vision Rückschritt und gefährdet somit die Herrschaftsfundamente. In diesem Sinne kommt der Ideologie die Aufgabe zu, die Menschen permanent für die Ziele des Regimes als Ausführungsorgan der weltanschaulichen Forderungen zu mobilisieren.

61 Vgl. exemplarisch Horst Adam, »Zur Entwicklung des Freund-Feind-Bildes im Staatsbürgerkundeunterricht der Klassen 7 und 8«, in: Geschichtsunterricht und Staatsbürgerkunde 2 (1970), S. 141-151.

62 Vgl. Hannah Arendt, Elemente und Ursprünge totaler Herrschaft. Antisemitismus, Imperialismus, Totalitarismus, 10. Aufl., Frankfurt a. M. 2005, S. 814. 
Ganz besonders deutlich lässt sich das machttaktische Moment anhand der verscbleiernden und kritikimmunisierenden Funktion (c3) verdeutlichen. Ideologien eignen sich vorzüglich dazu, reale Verhältnisse und Entwicklungen zu verschleiern und zu überdecken. Defizite politischen Handelns können jederzeit mit dem Verweis auf tiefere theoretische Einsichten und höher stehende Ziele, denen man zu dienen vorgibt, kaschiert werden. Falsches Handeln kann nach dieser Lesart als falsch durchgeführt, aber dennoch richtig gedacht verkauft werden. Der Glaube an den historisch vorbestimmten Endsieg verdeckte im Nationalsozialismus moralisch verwerfliche Kriegsziele und strategische Fehlentscheidungen ebenso, wie das Versprechen einer kommunistischen Gesellschaft den Blick auf eine desolate Wirtschaftspolitik und die Inhaftierung von „Republikfeinden" verstellte. In dieser Perspektive degeneriert die Ideologie tatsächlich zu einem bloß taktisch motivierten Machiavellismus. Die Machthaber finden sich in der angenehmen Lage wieder, gegen alle Kritik gefeit zu sein.

Bei jeder der drei hier genannten Funktionen steht die herrschaftsstabilisierende Dimension im Vordergrund: Die Erziehung und das Gefügigmachen von Menschen, die Mobilmachung für höhere Ziele sowie die Verschleierung politischer Fehlentscheidungen sind sämtlich in erster Linie auf die Konsolidierung und Prolongierung von Herrschaftsmacht gerichtet. Die konkrete empirische Ausgestaltung des Verhältnisses zwischen Überzeugungsglaube und Machtzynismus hängt realpolitisch maßgeblich vom Typus der politischen Führungsfiguren und der Rolle der Ideologen im jeweiligen ideokratischen Herrschaftsgefüge ab.

(d) Es fehlt noch eine letzte Funktion, die gleichsam quer zu allen bisher erarbeiteten Funktionen liegt bzw. in der sich alle anderen verdichten: die Legitimationsfunktion. Sie trifft den wesentlichen Aspekt ideokratischer Herrschaftsführung und fundiert daher gewissermaßen die übrigen acht Funktionen. Ideologisches Denken gerät zur Legitimationsstrategie politischen Handelns. Wie bereits ausgeführt, rechtfertigen ideokratische Herrschaftssysteme ihre Existenz auf der Grundlage ideologischer Dogmen, die die Geschichte oder ein göttliches Gesetz ihnen angeblich aufdiktiert habe und die sie einzulösen vorgeben. Aber nicht nur die bloße Existenz der Diktatur wird durch die Ideologie legitimiert, die jeweilige Weltanschauung liefert ebenfalls die Rechtfertigung für jedwedes politische Handeln und für alle moralisch verwerflichen Taten. ${ }^{63}$ In der Legitimationsfunktion fügen sich sämtliche überzeugungsbasierte und machttaktisch motivierte Funktionszusammenhänge zusammen. In diesem Sinne fasst Hans-Joachim Lieber den bereits erläuterten basalen Unterschied zwischen schlichten Autokratien und ideologiegeleiteten Diktaturen noch einmal zusammen: »Es wird nicht einfach schrankenlos Macht ausgeübt [...], sondern es wird schrankenlos ausgeübte Macht den Beherrschten gegenüber philosophisch gerechtfertigt. «64

Die Grundzüge des vorgestellten Funktionsmodells verstehen sich als Anknüpfungspunkte für eine generelle Theorie der Weltanschauungsdiktatur. Es kann sich hierbei nur

63 Vgl. Alexander Solschenizyn, Der Archipel Gulag, Band 1, Bern 1974, S. 172.

64 Hans-Joachim Lieber, Ideologie. Eine historisch-systematische Einführung, Paderborn et al. 1985, S. 109. 
um einige anschlussfähige Eckpunkte handeln, die für eine umfassende Theorie noch um weitere Aspekte ergänzt werden müssten.

\section{Die Aporie der Ideokratie}

Das Merkmal eines umfassenden Herrschaftsanspruchs, das viele Ideokratien kennzeichnet, soll nicht suggerieren, Ideokratien wären besonders langlebige Herrschaftsgebilde. Das Gegenteil ist der Fall: In der Regel verfügen sie über eine relativ kurze Verfallsdauer. Was sind die Gründe für die häufig relativ knapp bemessene Lebenserwartung von ideologiegeleiteten Diktaturen?

Zumeist scheitern Ideokratien an ihren selbstgesteckten Ansprüchen. Der Anspruch, die Gesellschaft umfassend zu durchherrschen, kann selten eingelöst werden, da dies quantitativ wie qualitativ hohe Anforderungen an die Herrschaftsorganisation stellt. Häufig kann die Einheit des Regimes nicht erhalten werden, da es nicht gelingt, die Menschen dauerhaft für das ideologische Ziel zu mobilisieren. Generationenwechsel bewirken in diesem Zusammenhang zumeist ein Erschlaffen ideokratischer Herrschaftsintensität. Ferner stehen die Machthaber in ideologiegeleiteten Diktaturen unter dem permanenten Druck, politische Problemlösungsstrategien in Zusammenhang mit den ideologischen Dogmen bringen zu müssen. Dadurch neigen solche Regime dazu, in der Herrschaftsausübung unflexibel und borniert zu werden. Je ideologisierter und dogmatischer eine Herrschaftsform ist, desto unausweichlicher wird es, Kompromisse und Zugeständnisse in der Herrschaftspraxis zu machen, womit Selbstwidersprüche nicht ausbleiben können. Welche Konsequenzen innere Selbstwidersprüche, herrschaftspraktische Borniertheit und ein Nachlassen der ideologischen Mobilisierung haben können, lässt sich anschaulich am Zerfall der Sowjetunion mit dem Machtantritt Michail Gorbatschows beobachten. ${ }^{65}$ Indem Gorbatschow dem Sowjetsystem die ideologische Grundlage entzog, verlor dieses mit der ideologischen Herrschaftslegitimation auch seine »raison d'être u und war somit dem Untergang geweiht. An diesem Beispiel lässt sich ablesen, wie fragil und verwundbar ideokratische Regime eigentlich sind. Sobald die ideologische Basis bröckelt, sehen sie sich in ihrer existentiellen Grundlage gefährdet.

In diesem Zusammenhang lässt sich ein zentraler Gedanke von Charles de Montesquieu (1689-1755) aus seinem Hauptwerk »De l’Esprit des lois« (1748) aufnehmen. Montesquieu hatte in seiner Herrschaftsformenlehre zwischen der Natur einer Regierung, also ihren Verfassungsstrukturen und dem Prinzip einer Regierung, d. i. das ihr zu Grunde liegende, das gesellschaftliche und politische Leben prägende Leitmotiv, unterschieden. ${ }^{66}$ Der Despotie als ihrer Natur nach gesetzes- und rechtloser Herrschaftsform hatte er das Prinzip der Angst zugeordnet. Allerdings widersprächen die Grunderfahrungen von Angst, Furcht und Ohnmacht der menschlichen Natur und aus diesem Grunde, so Montesquieu, trüge die Despotie den Keim ihres Untergangs von vorne her-

65 Vgl. hierzu ausführlich Vladislav M. Zubok, A Failed Empire. The Soviet Union and the Cold War from Stalin to Gorbatchev, North Carolina 2007, S. 303-335.

66 Vgl. Charles de Montesquieu, De L'Esprit des lois, avec les notes de l'auteur, Paris 1867, S. 18.

ZfP 58. Jg. 2/2011 
ein in sich. ${ }^{67}$ Diesen Gedanken etwas variierend lässt sich die Aussage treffen, dass auch Ideokratien schon von ihrer Natur her zum Scheitern verurteilt sind. Eine monistische Ideologie taugt nämlich im Kern nicht zur Legitimation von Herrschaft. Die monistische Grundstruktur von Ideologien mit ihrer Tendenz, die Welt als organische Einheit und als unteilbares Ganzes zu betrachten, muss sich mit innerer Notwendigkeit an der Komplexität, Pluralität und Unüberschaubarkeit der Verhältnisse brechen. Eugen Lemberg ist zuzustimmen, wenn er die grundsätzliche Aporie aller ideologischen Systeme im pluralistischen Wesen des Menschen und dem ihm inhärenten Forscherdrang sieht: Ein Gedankengebäude kann noch so schlüssig und konsistent auftreten, der Mensch wird von seiner Natur her stets neue Wege zur Wahrheit suchen. Und eben damit entreißt er geschlossenen Gedankensystemen und solchen Regimen, die auf ihnen aufbauen, den für sie überlebenswichtigen Boden. Wenn das ideologische System nicht mehr als Wahrheit anerkannt wird und als unwahr durchschaut ist, so verliert es seine Legitimationsgrundlage und hört unweigerlich auf zu bestehen. ${ }^{68}$ So gehen Herrschaftsintensität und Herrschaftsfragilität auf scheinbar paradoxe Weise eine untrennbare Verbindung ein. Diesen Befund gilt es sich auch in Zeiten neuer fundamentalistischer und terroristisch-islamistischer Tendenzen verhalten optimistisch immer wieder vor Augen zu führen.

\section{Zusammenfassung}

Der Aufsatz ist darum bemüht, einen Begriff zu rehabilitieren, den nahezu die gesamte Diktaturforschung im 20. Jahrhundert ignoriert und der in der aktuellen Debatte angesichts seines analytischen Potentials noch längst nicht die gebührende Berücksichtigung gefunden hat: die Ideokratie. Die Kernthese lautet, dass der Totalitarismus-Begriff nach wie vor für die vergleichende Untersuchung von Nationalsozialismus und Stalinismus ebenso wie auch in der Transformationsforschung eine ganz entscheidende Rolle spielt, dass allerdings der Ideokratie-Begriff für die Typenbildung in der Autokratieforschung methodisch besser geeignet ist. Dazu werden zunächst die Termini Ideologie und Ideokratie begriffsgeschichtlich eingeordnet und systematisiert. Anschließend werden das analytische Potential sowie einige wesentliche Vorzüge des Ideokratie-Konzeptes gegenüber der klassischen Totalitarismustheorie und anderen Ansätzen der vergleichenden Diktaturforschung herausgearbeitet. Danach wird der Ideokratie-Begriff mit Hilfe einer Funktionsanalyse zu einem forschungspraktischen Modell konzeptualisiert, bevor ein abschließender Blick auf die Grenzen und die Langlebigkeit ideologiegeleiteter Diktaturen geworfen wird.

\section{Summary}

The essay attempts to rehabilitate a term that has not only been largely neglected by modern dictatorship research, but also continues to be widely overlooked in current

67 Vgl. Montesquieu, ebd., S. 24-26.

68 Vgl. Lemberg, Ideologie und Gesellschaft, aaO. (FN 16), S. 54-56. 
debates: ideocracy. The main assumption is that the concept of totalitarianism still plays an important role in comparing National Socialism and Stalinism as well as for transformation research. However the term ideocracy seems to be better suited for autocracy typification. After a general historical overview, the terms ideology and ideocracy will be analytically explained. Subsequently, the analytical potential and the advantages of ideocracy vis-à-vis classic totalitarianism theory and other approaches to comparative studies of dictatorship will be examined. Thereafter, using a function analysis, a scientific model of ideocracy will be conceptualized, followed by a concluding view on the limits and durability of ideological dictatorships.

Manuel Becker, The Regime of Ideocracy. Re-examining the potential of a forgotten term in current dictatorship research

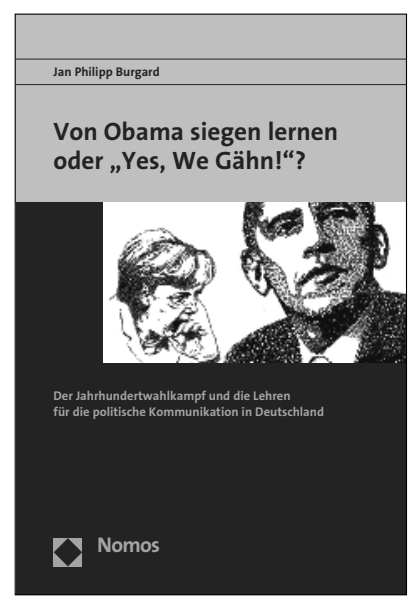

\section{Von Obama siegen lernen oder „Yes, We Gähn!"?}

Der Jahrhundertwahlkampf und die Lehren für die politische Kommunikation in Deutschland

Von Jan Philipp Burgard

2011, 256 S., geb., 49,-€

ISBN 978-3-8329-6670-6

nomos-shop.de/13696

Wie ist Barack Obama der kometenhafte Aufstieg vom unbekannten Senator zum ersten farbigen US-Präsidenten gelungen? Und was können deutsche Wahlkämpfer von ihm lernen?

„Allen, die wissen möchten, wie Wahlkämpfe aus ihrem lähmenden Dornröschenschlaf erwachen und zu spannenden Wettbewerben werden können, sei dieses Buch von Herzen empfohlen."

Tom Buhrow

Bitte bestellen Sie im Buchhandel oder

versandkostenfrei unter $\bullet$ www.nomos-shop.de

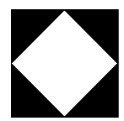

Nomos 